\title{
Study on Joint Driving Characteristics for Powered Exoskeleton Based on All-Joints Driving Model
}

\author{
Mingkui Zhang, Wenming Cheng, Fang Liu, and Huaixian Li \\ School of Mechanical Engineering, Southwest Jiaotong University, Chengdu 610031, China
}

\begin{abstract}
In order to study the changing characteristics of the driving torque, the driving power and the driving energy of all joints for the powered exoskeleton in the squat stance, multi-rigid-body system dynamics model of the powered exoskeleton was established based on the somatic structure characteristics parameters in the sagittal plane. The angular displacement motion equation of the ankle, the knee and the hip were obtained through the curves fitting method according to the data analysis and multi-visual machine motion measurement experiment of human-body movement. Dynamics explicit equation in matrix expression form of the powered exoskeleton multi-rigid-body system was deduced by selecting the angular displacement as generalization coordinates variable, and the equation of the driving power and the driving energy of all joints were established. The research shows that the amplitude of the angular displacement, angular velocity and angular acceleration of the knee is big, but that of the ankle and the hip is relatively small; the driving torque and the driving power of the knee is big and the rate of change is also very big, but those of the ankle and the hip are small; the driving energy of the knee is very big, and that of the ankle and the hip is small. Research results provide a theoretical basis for the design of the power system, the driving mode and control schemes for the powered exoskeleton.
\end{abstract}

KEYWORD: squat stance, powered exoskeleton, driving characteristic, motion characteristic.

\section{INTRODUCTION}

The powered exoskeleton is a kind of wearable personified and power-assisted system which can keep a highly consistent motion with human. During the motion process, the exoskeleton is highly coupled with human and can perceive the load from humanbody and its surroundings with sensors. The exoskeleton can make human carry greater load and improve the stamina of the wearer, and its real-time required power is provided by the control system and driving system [Dollar A M, 2008]. At present, many countries have developed the exoskeleton, such as BLEXX hybrid drive enhanced lower extremity exoskeleton [Zoss A, 2006], MIT passive exoskeleton [Walsh C J, 2007], HAL electric drive exoskeleton from University of Tsukuba [Kawabata T, 2009] and WSE lower extremity exoskeleton of Konya University [Onen U, 2014] and so on. In the domestic, Southwest Jiaotong University [LIU Fang, 2012], Southeast University [HAN Yali, 2015] and East China University of Science and Technology [CAO Heng, 2010] have launched related researches on the exoskeleton and developed experimental prototype in China, but in the aspect of the technology and market applications, there is still a large gap with foreign countries.

H.Kazerooni and R.Steger [Kazerooni H, 2006] thought that the powered exoskeleton is associated with four core technologies including novel control schemes, the lightweight design of the exoskeleton architecture, a body local area network to host the control algorithm and on-board power drive units. Among them, the key is to establish a kind of dynamic model which can be accurate under all kinds of postures and working conditions through theoretical and experimental methods and to design reasonable drive mode, novel control schemes and reliable drive system to achieve a real-time and effective control on the powered exoskeleton, helping it strengthen the carrying capacity and stamina of the wearer. H.Kazerooni [Racine J C, 2003] and J.C.Racine [JIA Shan, 2015] had analyzed the dynamics of the enhanced exoskeleton in the stance of swing, walking and jumping and so on, based on which the control algorithm and control schemes were designed. Through the CGA analysis and dynamics analysis, A.Zoss and H.Kazerooni [Zoss A, 
2006] obtained the driving torque and driving power of all joints of the exoskeleton and worked out rational drive model based on them. YL.Han [HAN Yali, 2015] had presented the actuator design of different joints and control schemes of different stances by analyzing the dynamics of the exoskeleton. Q.Guo [Guo Q, 2015] had established humanmachine coupling dynamics model based on Newton Method and carried out the design of control system and simulation calculation.

Powered exoskeleton has various motion stances and the working condition is different in different stances, which lead to different dynamics models, driving models and control schemes, therefore, the accurate dynamics analysis must be held on the powered exoskeleton in different kinds of stances and working conditions, especially in the extreme stance and special working conditions, to confirm the driving torque, driving power and driving energy of all joints, which provide the theoretical basis for the design of control system, power system and driving model. In this paper, aiming at the powered exoskeleton prototype, with the motion characteristics of all joints obtained from the somatic machine multi-vision motion capture experiment and data fitting, all joints' driving characteristics of the powered exoskeleton in the squat stance will be discussed.

\section{POWERED EXOSKELETON DYNAMICS MODEL CONSTRUCTION}

Joint driving characteristics $(d c)$ include joint driving torque $(d t)$, driving power $(d p)$ and driving energy $(d e)$. Assuming that there was no interaction force between the wearer and the powered exoskeleton, a dynamics model (showed as Fig.1 (A)) was established. $O_{1}, O_{2}$ and $O_{3}$ are respectively the ankle, the knee and the hip, which are connected by rotation pair. $l_{1}, l_{2}$ and $l_{3}$ are respectively the length of the calf, thigh and trunk. $S_{1}, S_{2}$ and $S_{3}$ are respectively the centroid of the calf, thigh and trunk. $l_{S 1}, l_{S 2}$ and $l_{S 3}$ are respectively the length from the centroid to its corresponding joint. $m_{1}, m_{2}$ and $m_{3}$ are the mass of the calf, thigh and trunk respectively. $\varphi_{1}, \varphi_{2}$ and $\varphi_{3}$ are the angle of the calf, thigh, trunk and $x$ axis respectively, which represent the angular displacement of each rod.

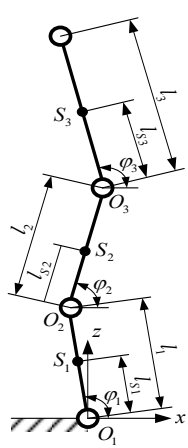

(A)

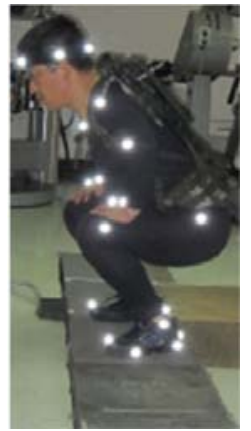

(B)
Fig.1 Dynamics model of powered exoskeleton (A) and experiment of data collecting (b) in squat

\subsection{Kinematics Analysis.}

The powered exoskeleton is a multi-rigid-body system with 3 degree of freedom (DOF).The angular displacement $\varphi_{1}, \varphi_{2}$ and $\varphi_{3}$ are selected as generalized coordinates $q_{1}, q_{2}$, and $q_{3}$, namely, $\varphi_{1}=q_{1}, \varphi 2=q_{2}$ and $\varphi 3=q_{3}$. Establishing fixed coordinate system $O_{1} x z,\left(x_{\mathrm{s} 1}, z_{\mathrm{s} 1}\right),\left(x_{\mathrm{s} 2}, z_{\mathrm{s} 2}\right)$ and $\left(x_{\mathrm{s} 3}, z_{\mathrm{s} 3}\right)$ are the coordinates of the $S_{1}, S_{2}$ and $S_{3}$ in the $O_{1} x z,\left(l_{s 1} \cos q_{1}\right.$, $\left.l_{s 1} \sin q_{1}\right), \quad\left(l_{1} \cos q_{1}+l_{s 2} \cos q_{2}, \quad l_{1} \sin q_{1}+l_{s 2} \sin q_{2}\right) \quad$ and $\left(l_{1} \cos q_{1}+l_{2} \cos q_{2}+l_{s 3} \cos q_{3}, l_{1} \sin q_{1}+l_{2} \sin q_{2}+l_{s 3} \sin q_{3}\right)$ respectively. The velocity and the angular velocity can be obtained by taking the first derivation of $\left(x_{s 1}\right.$, $\left.z_{s 1}\right),\left(x_{s 2}, z_{s 2}\right)$ and $\left(x_{s 3}, z_{s 3}\right)$ respectively.

\subsection{Kinetic Analysis.}

Lagrange dynamics equation of the powered exoskeleton multi-rigid-body system is described as

$$
\tau_{i}=\frac{\partial E_{p}}{\partial q_{i}}+\frac{d}{d t}\left(\frac{\partial E_{k}}{\partial \dot{q}_{i}}\right)-\frac{\partial E_{k}}{\partial q_{i}}, i=1,2,3
$$

The potential energy $E_{\mathrm{p}}$ and the kinetic energy $E_{\mathrm{k}}$ can be described as below:

$$
\begin{gathered}
E_{p}=\sum_{i=1}^{3} m_{i} z_{z_{i i}}=\left(\frac{1}{2} m_{1}+m_{2}+m_{3}\right) g l_{1} \sin q_{1}+\left(\frac{1}{2} m_{2}+m_{3}\right) g l_{2} \sin q_{2}+\left(\frac{1}{2} m_{3}\right) g l_{3} \sin q_{3} \\
\mathbf{E}_{\mathbf{k}}=\frac{1}{2}\left[\begin{array}{lll}
\dot{q}_{1} & \dot{q}_{2} & \dot{q}_{3}
\end{array}\right]\left[\begin{array}{ll}
J_{i j}
\end{array}\right]\left[\begin{array}{lll}
\dot{q}_{1} & \dot{q}_{2} & \dot{q}_{3}
\end{array}\right]^{\mathrm{T}}
\end{gathered}
$$

In Eq.(3), $J_{i j}(i=1,2,3 ; j=1,2,3)$ are equivalent rotational inertia and have something to do with dimension and mass distribution of the rod, which can be changed as the change of the component position and posture, and then they have an effect on the dynamics characteristics of the powered exoskeleton. They can be described as follows:

$\left\{\begin{array}{l}J_{11}=\left(\frac{1}{3} m_{1}+m_{2}+m_{3}\right) l_{1}^{2}, J_{22}=\left(\frac{1}{3} m_{2}+m_{3}\right) l_{2}^{2}, J_{23}=J_{32}=\frac{1}{2} m_{3} l_{2} l_{3} \cos \left(q_{2}-q_{3}\right) \\ J_{12}=J_{21}=\left(\frac{1}{2} m_{2}+m_{3}\right) l_{1} l_{2} \cos \left(q_{1}-q_{2}\right), J_{13}=J_{31}=\frac{1}{2} m_{3} l_{1} l_{3} \cos \left(q_{1}-q_{3}\right), J_{33}=\frac{1}{3} m_{3} l_{3}^{2}\end{array}\right.$

The second-order nonlinear differential Eq. (5) representing joint driving torque $\tau_{i}(i=1,2,3)$ can be obtained by putting Eq. (2) Eq.(4) into Eq. (1), as means antisymmetry, 


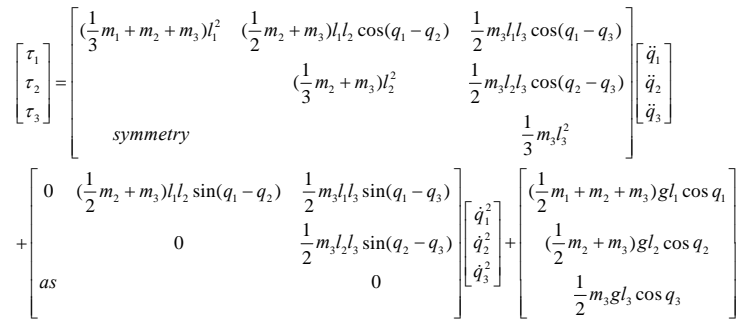

The joint driving power and driving energy of the powered exoskeleton is computed as follows:

$$
P_{i}=\left|\tau_{i} \dot{q}_{i}\right|, i=1,2,3
$$

\section{JOINT MOTION CHARACTERISTICS EXPERIMENT AND ANALYSIS}

Joint motion characteristics (mc) include joint angular displacement $(a d)$, angular velocity ( $a v)$ and angular acceleration $(a a)$. In order to get the joint motion characteristics, human-body data collecting experiment (Fig.1 (B)) in the squat stance was carried out. The load on the experimenter is $0 \mathrm{~kg}$. In the course of this experiment, the setup time is $3 \mathrm{~s}$, the squat time is $0.8 \mathrm{~s}$ and the stand adjustment time is 3 s. In order to reduce the error of data collecting due to equipment, experimenter and surroundings, repeated trails were completed. The trail equipment for data collecting is 6 cameras optical motion capture system (Qualisys AB, Sweden), and 3dimensional coordinate data of 23 reflective markers which include head, shoulders, chest, hips, knees, ankles, soles, heels and tiptoes was measured in this experiment. The sampling frequency is $200 \mathrm{~Hz}$.

In order to investigate the joint motion characteristics, the human body is simplified as multi-rigidbody system connected with multi-rigid bars. In the squat stance, because the motion of the right body is very similar to the left, it only needs to select the left body to investigate human joint motion characteristics. Joint angular displacement $(a d)$ curves (Fig.2A) were obtained through analyzing experimental data. Selecting the Fourier series as basis function, Levenberg-Marquardt (LM) nonlinear least squares method was used to fit discrete data of joint angular displacement, and the fitting equation (7) of joint angular displacement was got and the fitting error was small, which showed that joint angular displacement can be fitted by Fourier series.

$$
\varphi_{i}=\frac{a_{0}}{2}+\sum_{j=1}^{n}\left(a_{j} \cos j \omega t+b_{j} \sin j \omega t\right)
$$

In Eq.(7), $\varphi_{i}(i=1,2,3)$ are the angular displacement fitting equation of the ankle, knee and hip respectively. $n$ is fitting order, $\omega$ is angular frequency, $a_{0}, a_{j}$ and $b_{j}$ are constant coefficient. Angular velocity ( $\left.a v\right)$ curves (Fig.2B) and angular acceleration ( $a a$ ) curves (Fig.2C) of all joints are obtained by taking the first and second derivative of Eq. (7)respectively.
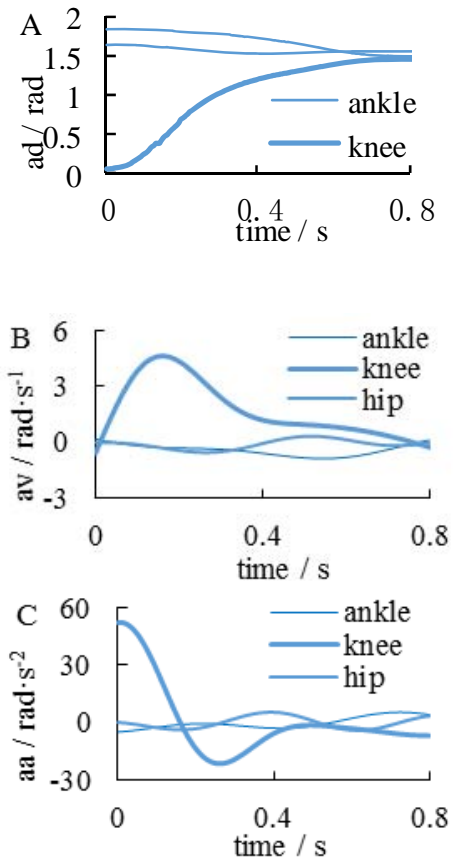

Fig.2 Joint motion characteristics curves

As shown in Fig.2, the amplitude of the angular displacement of the knee is big, but that of the ankle and the hip are relatively small, so the knee is the main motion joint and the ankle and hip are the auxiliary motion joint. In addition, the angular velocity and angular acceleration of the knee are large and have obvious changes in the first half, but they are small and their changes are smooth in the second half, while the angular velocity and angular acceleration of the ankle and hip are changing in the whole posture, but the changes are small.

\section{SIMULATION RESULTS}

The system structure parameters include $m_{1}=0.6 \mathrm{~kg}$, $m_{2}=0.9 \mathrm{~kg}, \quad m_{3}=8 \mathrm{~kg}, \quad l_{1}=0.364 \mathrm{~m}, \quad l_{2}=0.466 \mathrm{~m}$, $l_{3}=0.46$ and $t=0.8 \mathrm{~s}$. Because the joint motion characteristics between human and the powered exoskeleton are the same, the motion parameters of the powered exoskeleton can be deduced.

As shown in Fig.3, in the squat stance, the driving torque and driving power of the knee are very big and its rate of change is also big, but those of the ankle and hip are small, so the knee is the main driving joint and it requires highly sensitive driving system and control system, and the ankle and hip are the auxiliary driving joint. In addition, because the powered exoskeleton and human are highly coupled, in order to maintain a high degree of consistency and no interaction force between them, the sensing system must be highly sensitive to the force and torque from external environment exerted on the powered exoskeleton. 

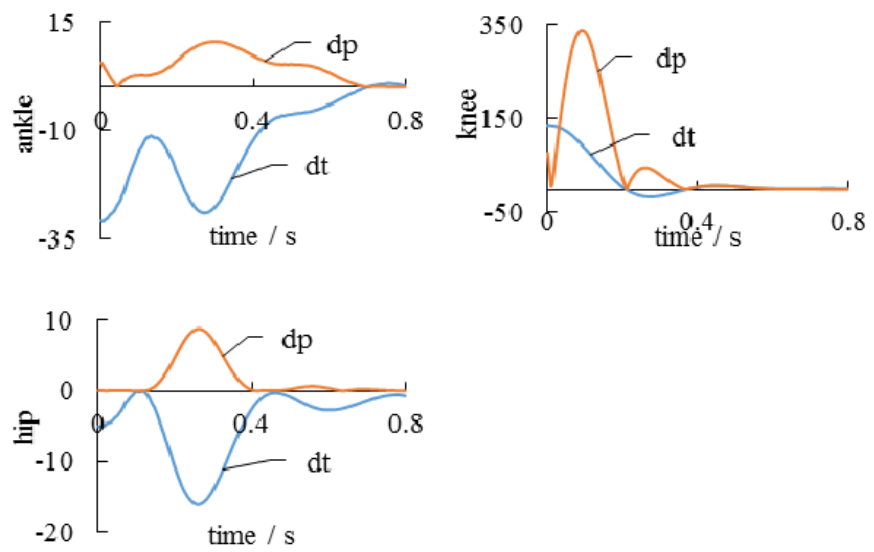

Fig.3 Joint driving torque (dt) and driving power (dp) curves
As shown in Table 1, the maximum driving torque of the knee (knee- $\tau_{\max }$ ) is as 4 times as the ankle- $\tau_{\max }$ and is as 8 times as the hip- $\tau_{\max }$, and the maximum driving power of the knee (knee- $P_{\max }$ ) is as nearly 30 times as the ankle- $P_{\max }$ and the hip- $P_{\max }$, and the rate of change of the knee- $\tau_{\max }$ and the knee- $P_{\max }$ is very big, so the drive and the control of the knee must have good stability and reliability and high sensitivity. In addition, the driving energy of the knee (knee$E$ ) is very large, about $45.89 \mathrm{~J}$, which accounts for $89.9 \%$ of the total energy ( $\left.E_{\text {total }}\right)$ of all joint. Because the power supply of the powered exoskeleton is onboard power supply, the power system of the knee requires energy optimization design and heat dissipation design and improves drive efficiency.

Table 1 Key value of joint driving characteristics

\begin{tabular}{|c|c|c|c|c|c|c|c|c|c|}
\hline & \multicolumn{9}{|c|}{ Key value } \\
\hline & $\begin{array}{l}\tau_{\max } / \\
\mathrm{Nm}\end{array}$ & $\begin{array}{l}\tau_{\min } / \\
\mathrm{Nm}\end{array}$ & $\begin{array}{l}\tau_{\text {avg }} / \\
\mathrm{Nm}\end{array}$ & $P_{\max } / \mathrm{W}$ & $P_{\text {ratio }}$ & $\begin{array}{l}P_{\text {avg }} / \\
\mathrm{W}\end{array}$ & $E / \mathrm{J}$ & $E_{\text {ratio }}$ & $\begin{array}{c}E_{\mathrm{to-}} \\
\mathrm{ta} / \mathrm{J}\end{array}$ \\
\hline ankle & 30.81 & 0.05 & 15.43 & 10.29 & $2.9 \%$ & 4.77 & 3.58 & $7.5 \%$ & \\
\hline knee & 134.31 & 0.14 & 67.23 & 337.06 & $94.5 \%$ & 57.36 & 45.89 & $89.9 \%$ & 50.77 \\
\hline hip & 16.06 & 0 & 8.03 & 8.75 & $2.6 \%$ & 1.62 & 1.3 & $2.6 \%$ & \\
\hline
\end{tabular}

The $\tau_{\max }$ and $P_{\max }$ of the ankle and hip is far less than those of the knee, and the $E_{\text {ratio }}$ of the ankle and hip are very small, so the ankle and hip can use passive drive [5] and non-power exoskeleton drive and so on. In future, the powered exoskeleton will face great challenge in research and design of the joint drive model, control schemes and power supply equipment and so on.

\section{SUMMARY}

In this paper, the motion characteristics of the human joint are analyzed through human body motion capture measurement experiment and data fitting. The driving characteristics of all joints are analyzed by establishing dynamics model of the powered exoskeleton in the squat stance. The main conclusions include as follows: (1) In the squat stance, joint angular displacement can be fitted by Fourier series, and the angular displacement, angular velocity and angular acceleration of the knee are big and the rate of change is also big, while those of the ankle and hip are relatively small. (2) The driving torque and driving power of the knee are very big and the rate of change is large, while those of the ankle and hip are small. (3) The driving energy of the knee is very big, accounting for $89.9 \%$ of the total energy of all joints, which shows that the power system of the knee requires energy optimization design and heat dissipation design and improves drive efficiency.

\section{REFERENCES}

CAO Heng, MENG Xianwei, LING Zhengyang, et al. Measurement system for plantar pressure of biped exoskeleton $\operatorname{robot}[\mathrm{J}]$. Chinese Journal of Senor and Actuators, 2010, 23(3): 326-330.

Dollar A M, Herr H. Lower Extremity Exoskeletons and Active Or thoses: Challenges and State-of-the-Art[J]. IEEE Transactions on Robotics, 2008, 24(1): 144-158.

Guo Q, Li S, Jiang D. A Lower Extremity Exoskeleton: Human-Machine Coupled Modeling, Robust Control Design, Simulation, and Overload-Carrying Experiment [J]. Mathematical Problems in Engineering. 2015, 2015: 1-15.

HAN Yali, YU Jianming, SONG Aiguo, et al. Parallel robot mechanism for ankle rehabilitation [J]. Journal of Southeast University (Natural Science Edition), 2015, 45(1): 45-50.

JIA Shan, WANG Xinsong, LU Xinliang, et al. Control of the exoskeleton's swing led based on the human-machine posture error at ankle joint [J]. Robot, 2015, 37(4):403-414.

Kawabata T, Satoh H, Sankai Y. Working Posture Control of Robot Suit HAL for Reducing Structural Stress [C]. IEEE International Conference on Robotics and Biomimetics (ROBIO), Guilin, China2009, 2009: 2013-2018.

Kazerooni H, Steger R. The Berkeley lower extremity exoskeleton [J]. Journal of dynamic systems, measurement, and control, 2006, 128(1): 14-25.

Kazerooni H, Racine J L, Huang L H, et al. On the control of the Berkeley Lower Extremity Exoskeleton (BLEEX) [C]. Proceedings. 2005 IEEE International Conference on Robotics and Automation (ICRA), Barcelona, Spain, April2005, 2005: 4353-4360.

LIU Fang, CHENG Wenming, ZHAO Nan. Study on stress testing to the mechanical structure of portable exoskeleton [J]. Journal of Mechanical Strength, 2012, 34(2): 198-202.

Onen U, Botsali F M, Kalyoncu M, et al. Design and Actuator Selection of a Lower Extremity Exoskeleton[J]. IEEE/ASME Transactions on Mechatronics. 2014, 19(2): 623-632. 
Racine J C. Control of a lower extremity exoskeleton for human performance amplification [D]. UNIVERSITY OF CALIFORAIN, BERKELEY, 2003.

Walsh C J, Endo K, Herr H. A quasi-passive leg exoskeleton for load-carrying augmentation [J]. INTERNATIONAL JOURNAL OF HUMANOID ROBOTICS. 2007, 4(3): 487506.

Zoss A, Kazerooni H. Design of an electrically actuated lower extremity exoskeleton[J]. ADVANCED ROBOTICS. 2006, 20(9): 967-988. 